\title{
Electromagnetic Compatibility Problem
}

National Cancer Institute

\section{Source}

National Cancer Institute. Electromagnetic Compatibility Problem. NCI Thesaurus. Code C63215.

Problem associated with the ability of a system to function in its electromagnetic environment without introducing intolerable disturbances to anything in its environment. 